\title{
THE ABSOLUTE MAGNITUDE AND STELLAR POPULATION OF A Bail STAR
}

\author{
R. D. MCCLURE* \\ Yale University Observatory, U.S.A.
}

\begin{abstract}
A star showing extreme features characteristic of the Ba II stars has been found in the old open cluster NGC 2420. A new colour-magnitude diagram has been constructed for the cluster, and a distance modulus of $11 \mathrm{~m} .7$ is obtained by fitting the unevolved main sequence to the zero-age main sequence. The location of the $\mathrm{Ba}$ II star is consistent with its being a disc population star of absolute magnitude $M_{v}=-0{ }^{\mathrm{m}} 3$, mass $1.4 M_{\odot}$ and age $2.4 \times 10^{9} \mathrm{yr}$.
\end{abstract}

The Ba II stars were first recognized as a class by Bidelman and Keenan (1951) because of the enhancement, on low dispersion spectra of features such as $\mathrm{CN}, \mathrm{CH}$, and $\mathrm{C}_{2}$ bands, and $\mathrm{Sr}$ II and $\mathrm{Ba}$ II lines. Normal luminosity indicators for $\mathrm{G}$ and $\mathrm{K}$ stars such as the $\lambda 4215 \mathrm{CN}$ band, and the $\lambda 4077$ line of $\mathrm{Sr}$ Il can have strengths similar to those in supergiant spectra, but Bidelman and Keenan recognized that other features suggest luminosities closer to giant stars. Warner (1965) and Eggen (1972) summarize the available data on individual $\mathrm{Ba}$ II stars for which some estimate of absolute magnitude can be obtained from $\mathbf{H}$ and $\mathrm{K}$ emission widths, trigonometric parallaxes etc. These estimates place the $\mathrm{Ba}$ II stars, on the average, slightly more luminous than the normal $\mathrm{G}$ and $\mathrm{K}$ giants of luminosity class III. A recent determination of the absolute magnitude of the $\mathrm{Ba}$ II stars as a class has been made by MacConnell et al. (1972) who obtained a mean magnitude of $M_{v}=-0.4$ from analysis of the proper motions of a sample of 120 stars, most of which have been discovered recently on Michigan objective prism plates. This determination again places the $\mathrm{Ba}$ II stars just slightly brighter than the normal $\mathrm{K}$ giants.

The origin of the peculiarities of the $\mathrm{Ba}$ II star spectra has been the subject of study of several authors who have carried out curve of growth analyses on high dispersion spectra of a few of the brighter examples. Burbidge and Burbidge (1957) and Warner (1965) conclude that carbon and the $\mathrm{S}$ process elements are overabundant. This indicates that heavy elements were synthesized in the interiors of the stars where the neutron capture process can occur, and in some way mixed to the surface in an advanced stage of evolution, perhaps during the carbon burning stage. Warner (1965) concludes that this process probably occurs in intermediate disc population stars of mass range $1-4 M_{\odot}$. Eggen (1972) estimates from the space motions of the Ba II stars, that they belong to the old disc population and have masses of 1 to $1.5 M_{\odot}$.

In order to determine an absolute magnitude more directly, and to pinpoint more accurately the type of stellar population to which a class of star belongs, it is always favourable to find a cluster that contains a star of the class. A Ba II star that, in fact, shows extreme enhancement of the features peculiar to this class has been found by

* Visiting Astronomer, Kitt Peak National Observatory which is operated by the Association of Universities for Research in Astronomy, Inc., under contract with the National Science Foundation. 
the author in the old open cluster NGC 2420. This is star ' $X$ ' in West's (1967) numbering system for the cluster. The spectrum of this star is similar to that of HD 24035 illustrated by MacConnell et al. (1972) in that the carbon features are stronger than in most Ba II stars and the $C_{2} \lambda 4737$ band is quite visible. The strength of the Ba II $\lambda 4554$ line would likely place the star in Warner's most extreme group (Ba 5).

Photographic photometry has been done on stars within a $5^{\prime}$ radius of the cluster center on $5 B$ and $4 \mathrm{~V}$ plates taken with the Yale 1-m telescope. A photoelectric sequence of 40 stars already existed for the cluster due to photometry by Arp (see West, 1967) and Sarma and Walker (1962). Twenty-four of these stars have been reobserved photoelectrically and two stars have been added to the faint end of the sequence from $U B V$ photometry on the Kitt Peak 50-in. telescope.

The resulting colour magnitude diagram is shown in Figure 1. The proper motion studies by Cannon and Lloyd (1970) and van Altena an Jones (1970) have been used

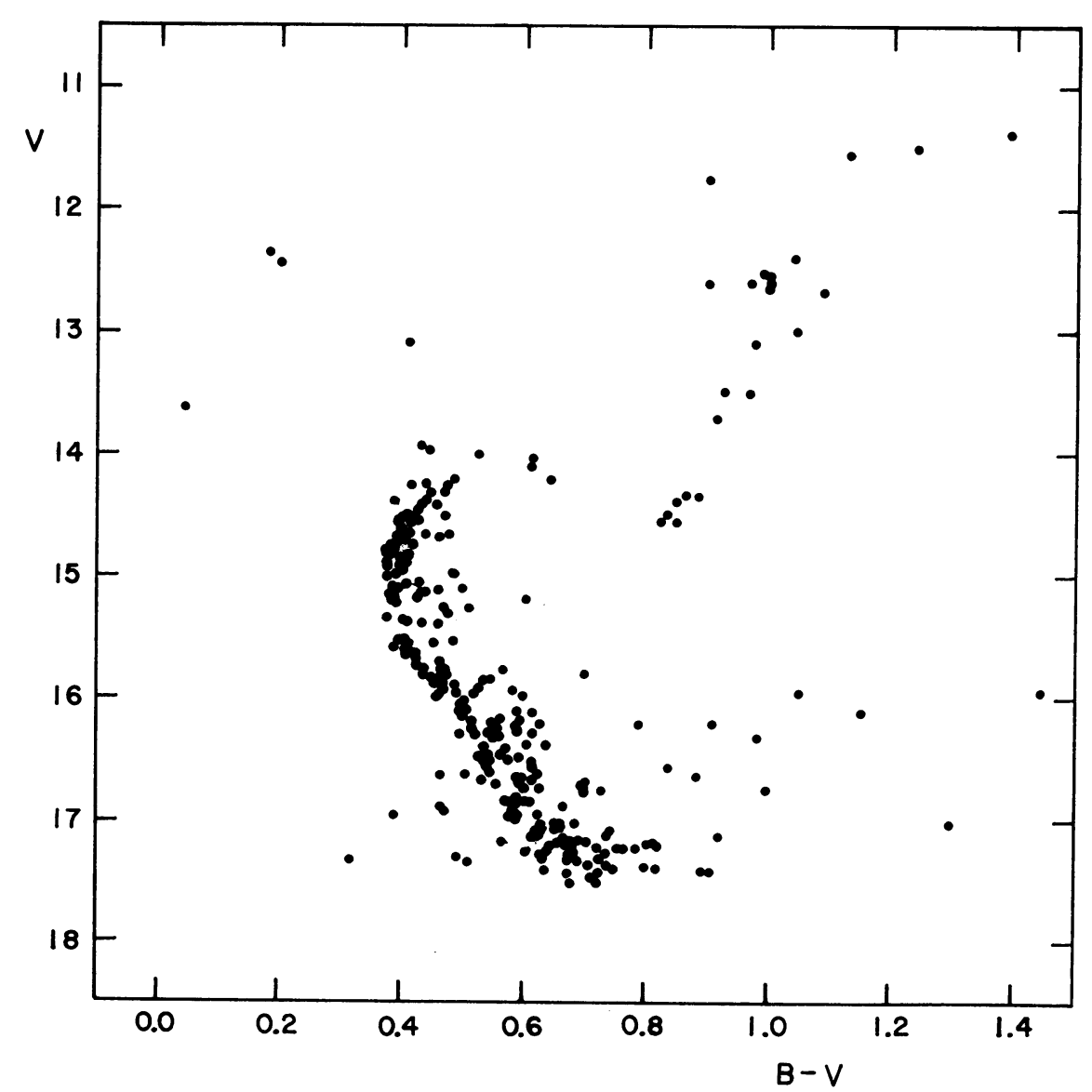

Fig. 1. The colour-magnitude diagram of the open cluster NGC $2420\left(l^{\mathrm{II}}=198^{\circ}, b^{1 \mathrm{I}}=20^{\circ}\right)$ from photographic photometry of 9 Yale 1-m telescope plates. The Ba II star is the brightest and reddest star on the diagram. 
to eliminate non-members. Cannon and Lloyd's plates extended to $\sim 15.7 \mathrm{~V}$ magnitude, so between this limit and the faint limit of the photoelectric sequence, all stars for which iris photometry could be done are plotted in Figure 1. The Ba II star is the brightest and reddest star on the diagram. There are three other bright stars in the same region of the diagram, and the bluest of these also appears to have peculiar line strengths. It is possible that it may be a marginal $\mathrm{Ba}$ II star similar to those listed in Table II of MacConnell et al. (1972). The Ba II line at 4554 is not visible in its spectrum although the $\mathrm{Sr}$ II $\lambda 4077$ line is very strong.

Star X, the extreme Ba II star has a proper motion that is close to the cluster proper motion but not quite close enough to say that it is a member on this basis. To check further on membership, spectra at $78 \AA \mathrm{mm}^{-1}$ were taken on the Kitt Peak 2-m telescope of 3 of the 4 brightest stars in Figure 1. The Ba II star velocity from the best plate agrees well with the velocity of the other two bright stars, both of which lie very close to the cluster center and are difinite members on the basis of proper motion. The velocities of these stars fall within the range $86-96 \mathrm{~km} \mathrm{~s}^{-1}$. With such a large velocity, this low-spread provides a high probability that the Ba II star is a member. A second poorer exposure of the Ba II spectrum gives a velocity of $134 \mathrm{~km} \mathrm{~s}^{-1}$, but this higher value may be due to the quality of the plate. The mean error in velocity per plate, however, is only $\sim 5 \mathrm{~km} \mathrm{~s}^{-1}$ so the possibility exists that there is a true variation.

Arp's photoelectric sequence revealed a rather large ultraviolet excess for an open cluster; $\delta(U-B)=+0^{\mathrm{m}} 09$. Photoelectric photometry by the author confirms this large excess, and in addition narrow band photometry on the DDO photometric system (defined by McClure and van den Bergh, 1968) shows that the $\mathrm{K}$ stars in the clump of the giant branch have weak $\mathrm{CN}$ bands, such as one would expect for metal weak stars.

West (1967) has discussed the reddening of the cluster and concludes on the basis of the $U B V$ photometry of the photoelectric sequence stars that the reddening is very small. He uses a value of $E(B-V)=0 \mathrm{~m} .01$. The reddening can also be determined from DDO photometry using the method described by McClure and Racine (1969). Again, the reddening appears to be negligible, and no reddening corrections have been applied here.

In order to obtain a distance for the cluster, an attempt has been made to fit the main sequence to the zero-age main sequence (Table 1 of Eggen, 1965). Corrections have been made to the main sequence for line blanketing effects suggested by Wildey et al. (1962) for an ultraviolet excess of $\delta(U-B)_{0.6}=+0.09$. After these corrections the main sequence fitting results in a distance modulus of 11.7 for the cluster. This places the cluster at a distance of $2200 \mathrm{pc}$, and because of its high galactic latitude of $20^{\circ}$ it is located more than $800 \mathrm{pc}$ above the galactic plane. It was this large distance from the plane that led van den Bergh (1958) to conclude that this was probably a very old cluster.

The high galactic latitude and remoteness of the cluster provides further evidence in favour of the membership of the Ba II star. MacConnell et al. (1972) estimate that about $1 \%$ of $\mathrm{G}$ and $\mathrm{K}$ giants in the solar neighborhood are $\mathrm{Ba}$ II stars. In order to investigate the probability of finding a red giant star of this brightness within the area 
of the cluster, iris photometry was done (by Mr T. Forrester) on a field centered 0.5 south of the cluster, and of area 1.5 times the area of the cluster photometry. No stars were found in this area with $B-V>0 \mathrm{~m} .70$ and $V<12 \mathrm{~m} .9$. Combining this fact with the estimate that $1 \%$ of $\mathrm{G}$ and $\mathrm{K}$ giants are $\mathrm{Ba}$ II stars leads to the conclusion that the probability of star $\mathrm{X}$ being a field star is negligible.

The derived distance modulus of the cluster places the Ba II star at an absolute magnitude of $M_{v}=-0.3$, in fortuitous agreement with the mean magnitude derived by MacConnell et al. (1972) for this class. The NGC 2420 colour-magnitude diagram can be converted to the $\log L$ vs $\log T_{\text {eff }}$ plane and fitted to the theoretical isochrones computed recently by Demarque and Gisler (1972). This results in an age determination of $2.4 \times 10^{9} \mathrm{yr}$ (assuming a chemical composition of $Y=0.25$ and $Z=0.01$ ) and a main sequence turn-off mass of $1.4 M_{\odot}$. This appears to confirm Eggen's (1972) conclusion that the $\mathrm{Ba}$ II stars are old disc stars of 1 to $1.5 M_{\odot}$.

\section{Acknowledgements}

The author wishes to thank Dr Pierre Demarque for fitting the cluster to the theoretical isochrones and for many inspiring discussions.

\section{References}

Altena, W. F. van and Jones, B. F.: 1970, Astron. Astrophys. 8, 112.

Bergh, S. van den: 1958, Z. Astrophys. 46, 176.

Bidelman, W. P. and Keenan, P. C.: 1951, Astrophys. J. 114, 473.

Burbidge, E. M. and Burbidge, G. R.: 1957, Astrophys. J. 126, 357.

Cannon, R. D. and Lloyd, C.: 1970, Monthly Notices Roy. Astron. Soc. 150, 279.

Demarque, P. and Gisler, G.: 1972, Bull. Am. Astron. Soc. 4, 326.

Eggen, O. J.: 1972, Monthly Notices Roy. Astron. Soc. 159, 403.

MacConnell, D. J., Frye, R. L., and Upgren, A. R.: 1972, Astron. J. 77, 384.

McClure, R. D. and Bergh, S. van den: 1968, Astron. J. 73, 313.

McClure, R. D. and Racine, R.: 1969, Astron. J. 74, 1000.

Sarma, M. K. S. and Walker, M. F.: 1962, Astrophys. J. 135, 11.

Warner, B.: 1965, Monthly Notices Roy. Astron. Soc. 129, 263.

West, F. R.: 1967, Astrophys. J. Suppl. 14, 384.

\section{DISCUSSION}

Keenan: The recognition of a barium star in a cluster and its luminosity classification are very exciting! Does it not appear now that there is a group of these barium-carbon stars?

McClure: Yes. Perhaps all old disc stars go through this stage in their evolution. 